\section{A0043 Prognostic Value of Serially Estimated Serum Procalcitonin Levels in Traumatic Brain Injury Patients with or without Extracranial Injury: A Longitudinal Observational Study \\ Gaurav S. Tomar, ${ }^{1}$ Keshav Goyal, ${ }^{2}$ Gyaninder P. Singh, ${ }^{2}$ Shweta Kedia, ${ }^{3}$ Arvind Chaturvedi ${ }^{2}$ \\ ${ }^{1}$ Department of Neuroanaesthesiology and Critical care, Medanta the Medicity Hospital, Gurugram, Haryana, India 2Department of Neuroanaesthesiology and Critical care, All India Institute of Medical Sciences, New Delhi, India ${ }^{3}$ Department of Neurosurgery, All India Institute of Medical Sciences, New Delhi, India}

Background: Traumatic brain injury (TBI) is associated with a majority of trauma deaths, and objective tools are required to understand the severity of an injury. The application of serum markers like procalcitonin (PCT) for TBI may assess the severity and thus aid in prognostication as well as correlation with mortality and outcome.

The primary objective was to determine the correlation between procalcitonin concentrations with the outcome at discharge or mortality. Secondary objectives were to evaluate correlation with associated extracranial injuries and complications during hospital stay.

Materials and Methods: In total, 186 TBI patients aged $>18$ years with minimum survival for at least 12 hours admitted to the neuro ICU at the level 1 trauma center were prospectively included in the study and divided into two groups: TBI with and without extracranial injuries. All admitted patients were treated according to the institutional protocols and accepted standards of care. PCT values (on days 1,2 , and 5 after trauma), all clinical, microbiological, laboratory data along with all diagnostic and therapeutic interventions with the duration of treatment in the ICU were noted.

Results: Median PCT level at admission, day 2 and day 5 in TBI patients with extracranial injuries were $3.0,0.83$, and $0.69 \mathrm{ng} / \mathrm{mL}$. Regression analysis of primary mortality (due to TBI) with PCT cutoff values at admission $(>5.5 \mathrm{ng} / \mathrm{mL})$ and on day 2 (> $1.16 \mathrm{ng} / \mathrm{mL}$ ). But for secondary mortality (due to complications of TBI), PCT cutoff values at admission $(>2.17$ $\mathrm{ng} / \mathrm{mL})$, on day $2(>1.16 \mathrm{ng} / \mathrm{mL})$, and on day $5(>1.2 \mathrm{ng} / \mathrm{mL})$ were significant. However, for primarily CNS cause of mortality, PCT cutoff level more than $5.5 \mathrm{ng} / \mathrm{mL}$ carried maximum AUC of $75 \%$, and for secondary cause (sepsis) of mortality, cutoff values more than $1.2 \mathrm{ng} / \mathrm{mL}$ had sensitivity or specificity of $68 \%$.

Conclusions: This observational study demonstrates the relation between procalcitonin concentrations with the outcome at days 1,2 , and 5 post-injury. PCT level estimation can be an indispensable tool for outcome assessment in TBI patients with or without extracranial injuries and its level can independently predict poor prognosis either based on the severity of CNS injury or secondary complications in initial 2 to 3 days.

\section{A0044 Encephalitis: An "Enigma” \\ Abhijeet Raha ${ }^{1}$ \\ ${ }^{1}$ Department of Anaesthesia, Rahman Hospitals Pvt. Ltd., Guwahati, Assam, India}

Background: Encephalitis is an acute inflammation of the brain parenchyma with varied etiologies that are difficult to diagnose. In majority of the cases, acute encephalitis is infective in origin, and less commonly, it may be autoimmune. Here we report a perplexing case of acute encephalitis.

Case Description: A 55-year-old male entomologist presented in emergency department with chief complaints of pain in the right knee, urinary retention, and constipation. He had no other known comorbidities. Baseline vitals and routine laboratories were unremarkable. T2 screening of the brain was normal, and MRI spine showed no significant compressive/noncompressive myelopathy. On the day 4 of hospital admission, the patient became drowsy for which he was intubated and kept on mechanical ventilation. Serum electrolytes and liver functions were normal, but serum ammonia was marginally raised $(49.1 \mu \mathrm{mol} / \mathrm{L})$. Metabolic encephalopathy was suspected, and the patient was started on lactulose and rifaximin. CSF analysis showed raised proteins, pleocytosis, and predominant lymphocytes, indicating viral/tubercular meningoencephalitis. On this ground, intravenous methyl prednisolone and antitubercular (ATT) drugs were started. Patient remained deeply comatose with GCS of E1VTM1. Electroencephalogram (EEG) ruled out nonconvulsive status epilepticus. Various reports including CSF TB-PCR, vasculitis profile, HSV IgG/IgM, Japanese encephalitis, NMDA $\mathrm{Ag}$, and VGKC Ag all were inconclusive. After these investigations, ATT was stopped.

Repeat brain MRI revealed T2 hyperintense signals with mild restricted diffusion in bilateral parahippocampal gyri, right insular cortex, left basal ganglia, and bilateral temporoparietal lobes in cortical, subcortical locations, bilateral cerebral peduncles, mid-brain, pons, medulla, and cervical cord. A differential diagnosis of acute disseminated encephalomyelitis was suspected. Patient received IV methyl prednisolone $1 \mathrm{~g}$ /day for 5 days and then tapered off. By day 10 in ICU, patient's GCS started improving and he was discharged from the ICU on the 30th ICU day with E4V5M6 status. His power in both upper limbs was $4 / 5$, proximal weakness more than distal and in both lower limbs was $2 / 5$.

Conclusions: There are various etiologies of encephalitis. Clinical presentation, course, and outcome may be quite perplexing in uncommon etiologies. Autoimmune encephalitis has been shown to have favorable outcome over viral etiology. As a diagnosis of exclusion and owing to its clinical course, we made a provisional diagnosis of autoimmune encephalitis. 